\title{
Abordagem e complicações medicamentosas na tontura
}

\author{
Patrícia Mano ${ }^{*}$
}

\begin{abstract}
Resumo
As medicações nas vestibulopatias são uma terapia de apoio, e não a solução isolada para a tontura do paciente. Na escolha do tratamento medicamentoso a ser utilizado no paciente com tontura, é importante que levemos em consideração a patologia de base e seu curso natural, as comorbidades do paciente e a necessidade de manter suas atividades durante o período de recuperação. Não existe a medicação perfeita, mas sim a melhor droga a ser utilizada naquele momento para aquele paciente. O objetivo do tratamento durante a crise labiríntica é reduzir o sintoma de vertigem, bem como as náuseas e vômitos experimentados pelos pacientes, o mais rapidamente possível. Existem diversas classes de medicações que são utilizadas para esta finalidade, sendo os anti-histamínicos, anticolinérgicos e benzodiazepínicos as mais frequentemente utilizadas. Estados crônicos de desequilíbrio geralmente têm como sintoma tonturas não rotatórias. Neste momento, a principal maneira de tratamento é a correção dos fatores etiológicos.
\end{abstract}

Descritores: Anormalidades induzidas por medicamentos; Tontura; Polimedicação.

\section{Abstract \\ Approach to and drug complications in dizziness}

The medications in the labyrinth diseases are a support therapy, and not an isolated solution for the patient's giddiness. To choose the drug treatment that will be used in the patient with dizziness, it is important that let us take in consideration the pathology of base and its natural course, the comorbidities of the patient and the necessity to keep its activities during the period of recovery. The perfect medication does not exist, but yes the best drug to be used at that moment for that patient. The objective of the treatment during the labyrinth crisis is to reduce the vertigo symptom, as well as nauseas and vomits tried for the patients, more quickly possible. Diverse medication classrooms exist that are used for this purpose, having been antihistamines, anticholinergics and benzodiazepines more frequent used. Chronic states of disequilibrium generally have as symptom not rotatory giddiness. At this moment, the main way of treatment is the correction of the etiological factors.

Keywords: Abnormalities, drug-induced; Dizziness; Polyfarmacy.
1. Departamento de Otorrinolaringologia. Hospital das Clínicas. Faculdade de Medicina. Universidade de São Paulo. São Paulo, SP, Brasil.

*Endereço para correspondência:

Departamento de Otorrinolaringologia, Hospital das Clínicas, Faculdade de Medicina, USP

Av. Doutor Enéias de Carvalho Aguiar, 255, 60 andar, sala 61-67 São Paulo, SP, Brasil. CEP: 05403-000.

E-mail: drapatriciamano@gmail.com

Revista HUPE, Rio de Janeiro, 2015;14(1):37-41

doi: 10.12957/rhupe.2015.14996

Recebido em 28/07/2014. Aprovado em 01/10/2014.

\section{Resumen}

\section{Enfoque y complicaciones medicamentosas en el mareo}

Las medicamentos en las vestibulopatías son una terapia de apoyo, y no la única solución para el mareo del paciente. En la elección del tratamiento medicamentoso que debe usarse en el paciente con mareo, es importante tener en cuenta la patología de base y su curso natural, las comorbilidades del paciente y la necesidad de mantener sus actividades durante el período de recuperación. No existe medicamento perfecto, pero si el mejor que debe utilizarse para el paciente en ese momento. El objetivo del tratamiento durante la crisis laberíntica es reducir el síntoma de vértigo, tanto como las náuseas y vómitos experimentados por los pacientes, tan pronto como sea posible. Hay varias clases de fármacos que se utilizan para este propósito, siendo los antihistamínicos, anticolinérgicos y benzodiazepínicos los más frecuentemente utilizados. Estados crónicos de desequilibrio generalmente tienen como síntoma mareos no rotatorios. En este momento, la principal forma de tratamiento es la corrección de los factores etiológicos.

Palabras clave: Anomalías inducidas por medicamentos; Mareo; Polifarmacia. 


\section{Artigo de revisão}

\section{Introdução}

Dentre a gama de terapias que podemos instituir no paciente com tontura, o uso de medicações nos ajuda a minimizar os efeitos neurovegetativos e a acelerar a compensação vestibular. Entretanto, o sucesso terapêutico depende primordialmente da obtenção de um diagnóstico adequado, pois só assim conseguiremos corrigir ou eliminar as patologias de base. As medicações nas vestibulopatias são, portanto, uma terapia de apoio, e não a solução isolada para a tontura do paciente.

O labirinto posterior é um sistema sensitivo periférico que capta as informações de movimento e as encaminha para as vias neuronais envolvidas com o equilíbrio corporal. Essa transmissão é mediada por neurotransmissores, que podem ter características excitatórias ou inibitórias, dependendo do local de atuação.

O quadro a seguir aponta os principais neurotransmissores do sistema vestibular, sua função e locais de atuação.

Na escolha do tratamento medicamentoso a ser utilizado no paciente com tontura, é importante que levemos em consideração a patologia de base e seu curso natural, as comorbidades do paciente e sua necessidade de manter suas atividades durante o período de recuperação. Não existe a medicação perfeita, mas sim a melhor droga a ser utilizada naquele momento, ponderando todos esses fatores.

Após uma lesão vestibular unilateral, ocorre uma assimetria de informação que chega ao sistema nervoso central, ocasionando, como resposta cerebelar e cortical, uma inibição do labirinto não lesado, na tentativa de manter a informação o mais simétrica possível. Com o passar dos minutos, há o início de toda uma modificação plástica dos receptores de neurotransmissores, para que ocorra uma compensação. De uma maneira geral, portanto, o objetivo do tratamento numa fase aguda é realizar a sedação labiríntica, e assim reduzir a assimetria de informações e o mal-estar do paciente, devendo esta ser mantida pelo menor tempo possível, a fim de que a compensação central seja estimulada.

\section{Tratamento na fase aguda}

O objetivo do tratamento durante a crise labiríntica é reduzir o sintoma de vertigem, bem como as náuseas e vômitos experimentados pelos pacientes, o mais rapidamente possível. Nesse sentido, a via de administração endovenosa é preferencial, sendo os supressores vestibulares e antieméticos as drogas mais comumente utilizadas.

Os supressores vestibulares atuam reduzindo a transmissão de sinais do núcleo vestibular para o centro regulador do vômito e outros sistemas autonômicos centrais. Tipicamente, tais drogas agem em mais de um neurotransmissor, os quais também não são específicos do sistema vestibular, promovendo uma intensa gama de efeitos colaterais e adversos.

É importante mantermos em mente que o uso de

Quadro 1. Principais neurotransmissores do sistema vestibular, sua função e locais de atuação.

\begin{tabular}{|c|c|c|}
\hline Neurotransmissor & Função & Local de atuação \\
\hline Ácido gama-aminobutírico (GABA) & Inibidor & $\begin{array}{l}\text { Células ciliadas, núcleos vestibulares, vias vestíbu- } \\
\text { lo-oculares, vias vestibuloespinhais e cerebelo }\end{array}$ \\
\hline Glutamato & Excitatório & Células ciliadas, vias vestíbulo-oculares \\
\hline Acetilcolina & Excitatória & $\begin{array}{l}\text { Células ciliadas, núcleos vestibulares, vias vestibu- } \\
\text { loespinhais }\end{array}$ \\
\hline Histamina & Inibitória & Núcleos vestibulares \\
\hline Glicina & Inibitória & $\begin{array}{l}\text { Núcleos vestibulares, vias vestibuloespinhais, vias } \\
\text { vestíbulo-oculares, cerebelo }\end{array}$ \\
\hline Dopamina & Excitatória & Zona quimiorreceptora do bulbo \\
\hline Serotonina & Modulação & $\begin{array}{l}\text { Transmite sinais vagais do trato gastrointestinal para } \\
\text { o centro emético, além de modular positivamente a } \\
\text { atividade dos neurônios vestibulares centrais }\end{array}$ \\
\hline
\end{tabular}


tais medicações deve ser restrito às tonturas rotatórias - ou seja, de origem vestibular - e pelo menor tempo possível, visto que dificultam o processo natural de compensação central, e podem levar a quedas nos pacientes com desequilíbrio por outras patologias devido à sedação desencadeada. Além disso, tratamentos profiláticos com uso prolongado de supressores vestibulares devem ser evitados em virtude da tolerância e da dependência que provocam.

Existem diversas classes de medicações que são utilizadas para esta finalidade, sendo os antihistamínicos, anticolinérgicos e benzodiazepínicos as mais frequentemente utilizadas. Devido ao alto grau de sedação, pacientes devem ser afastados de atividades que necessitem de bastante alerta, e devemos ter cautela caso o paciente já faça uso de drogas depressoras do sistema nervoso central ou álcool.

\section{Tratamento de fase crônica ou manutenção}

Estados crônicos de desequilíbrio geralmente têm como sintoma tonturas não rotatórias. Neste momento, a principal maneira de tratamento é a correção dos fatores etiológicos que impedem que as informações cheguem adequadamente ao núcleo vestibular. $\mathrm{O}$ diagnóstico otoneurológico adequado é fundamental para o sucesso terapêutico nesta fase. Dentre a série de medicações que podemos utilizar, as mais comuns são os vasodiladores, os histaminérgicos, os hemoreológicos e os antidepressivos.

\section{Principais classes de drogas em otoneurologia}

Anticolinérgicos: promovem redução da excitação causada pela acetilcolina, sendo, portanto, supressor vestibular. O exemplo clássico é o patch transdérmico de escopolamina - bastante utilizado por pacientes portadores de cinetose, em viagens de barco -, porém este não é comercializado no Brasil. Ainda assim, esta classe de medicação deve ser bem estudada em otoneurologia, visto que diversas drogas apresentam a ação anticolinérgica como efeito adicional. Seu uso é contraindicado nos pacientes portadores de glaucoma, obstrução pilórica ou intestinal e bexiga neurogênica. Na forma de patch, não é recomendada para crianças, pois não se sabe a dose exata que será liberada, podendo gerar muitos efeitos colaterais. Pode levar a alterações de memória, alucinações e confusão mental, devendo ser evitada em idosos. Ainda, pode provocar alteração do reflexo de acomodação visual, boca seca, miose, retenção urinária aguda e irritação local no uso do patch.

Anti-histamínicos: reduzem a atividade neural vestibular, atuando como sedativo desde as células ciliadas até os núcleos vestibulares. Todos os três receptores de histamina (H1, H2 e H3) são expressos no núcleo vestibular e em sua periferia, sendo H1 e H2 pós-sinápticos e o H3 pré-sináptico. Os receptores H3 modulam a ação do GABA no núcleo vestibular. A maioria dos antihistamínicos usados como supressores vestibulares são bloqueadores H1 e têm também ação anticolinérgica. São eles o dimenidrinato, a meclizina e a prometazina. Seu principal efeito colateral é a sonolência. Não devem ser usados em asmáticos, portadores de enfisema, doença pulmonar obstrutiva crônica (DPOC) ou hiperplasia prostática benigna. A meclizina e o dimenidrinato são considerados seguros para uso em grávidas.

Benzodiazepínicos: são agonistas do GABA, o principal inibidor dos sistemas vestibular periférico e central. São bastante efetivos no controle da ansiedade relacionada à tontura. Podemos citar como exemplo, neste grupo, o diazepan, o clonazepan, o bromazepan, o alprazolan e o piracetan - o qual é um derivado cíclico do GABA. Caso sejam utilizados por longos períodos, deverão ser retirados lentamente, visto que a suspensão abrupta desses medicamentos leva a sintomas que simulam novo evento labiríntico e, com isso, o paciente pode apresentar cefaleia, vômitos, náusea e tontura. É importante ressaltar que o diazepan está contraindicado no primeiro trimestre de gestação pelo risco de malformação fetal e também em lactantes, pois o metabolismo deste remédio é reduzido em neonatos, podendo ocasionar a toxicidade do bebê. Promovem sedação, prejuízo de memória, aumento do risco de quedas, tolerância e dependência.

Bloqueadores dos canais de cálcio: possuem ação supressora vestibular, tendo atuação anticolinérgica, anti-histamínica e bloqueadora dopaminérgica. Promovem regulação da homeostase do cálcio na orelha interna e vasodilatação secundária à sua ação na musculatura lisa dos vasos, promovendo maior aporte de oxigênio e glicose. São eles a cinarizina, a flunarizina e a nimodipina. Predispõem ao parkinsonismo, tremores, depressão, obesidade, constipação, boca seca e distúrbios de metabolismo de glicose, devendo ser evitados em idosos e crianças. É benéfico nas enxaquecas.

Antieméticos: promovem a redução das náuseas e vômitos por ação central ou diretamente gastrointes- 


\section{Artigo de revisão}

tinal, dependendo da classe de droga. Existem diversos neurotransmissores envolvidos no mecanismo de êmese, sendo os principais a dopamina, a histamina, a acetilcolina e a serotonina. Dentre os bloqueadores dopaminérgicos, temos a metoclopramida, a prometazina e a domperidona que, além do bloqueio da dopamina - neurotransmissor excitatório central -, promovem sedação vestibular central por efeito anticolinérgico e também efeito vasodilatador devido ao bloqueio alfa-adrenérgico. Apresentam como efeitos colaterais a sonolência, efeitos endócrinos e extrapiramidais, mais frequentes em crianças e idosos. Os inibidores de dopamina devem ser administrados com cautela em pacientes renais crônicos.

Vasodilatadores: favorecem o aporte de glicose e oxigênio por meio do relaxamento da musculatura lisa da parede dos vasos, atuando principalmente na microcirculação. Podem ter ação direta, como a papaverina, o ácido nicotínico, a nicergolina, o carbogênio e a histamina (por mecanismo secundário), ou ação indireta, bloqueando a ação das catecolaminas e inibindo a vasoconstricção. Este últimos são exemplificados pela diidroergocristina e os bloqueadores dopaminérgicos, conforme citado anteriormente. Os efeitos colaterais mais frequentes são rubor facial e hipotensão postural.

Anticonvulsivantes: são sedativos vestibulares. A carbamazepina inibe as descargas neuronais repetitivas e reduz a propagação sináptica dos impulsos excitatórios em neurônios despolarizados por meio do bloqueio dos canais de sódio. A gabapentina promove aumento do GABA no sistema nervoso central, redução do glutamato e bloqueio dos canais de sódio e cálcio nos neurônios. $\mathrm{O}$ ácido valproico age bloqueando os canais de sódio e inibindo a GABA-transaminase, ação esta que promove acúmulo do GABA na fenda sináptica. Como efeitos colaterais, podem ocorrer náuseas, vômitos, anorexia, sonolência, ataxia e tremores. $\mathrm{O}$ ácido valproico pode levar a aumento das enzimas hepáticas e a carbamazepina tem como efeito adverso a toxicidade hematológica, reações de hipersensibilidade, diplopia e visão turva.

Histaminérgicos: seu principal representante é a betahistina, um análogo da L-histidina, precursor da histamina. Através de sua ação antagonista do receptor H3 nos neurônios pré-efetores, promove aumento da síntese de histamina nos núcleos vestibulares, reduzindo a descarga de repouso da ampola e estimulando o fluxo coclear na estria vascular. Promove ainda aumento do fluxo vertebrobasilar, melhorando a oxigenação coclear. Os principais efeitos colaterais são broncoespasmo, perturbações gastrointestinais, cefaleia e rash cutâneo. É considerada uma droga facilitadora da compensação central e promove significativa melhora na doença de Meniere.

Hemorreológicos: são consideradas drogas facilitadoras da compensação central. Promovem modulação do fluxo na microcirculação, aumentando a irrigação tissular e permeabilidade capilar. Diminuem a viscosidade do sangue e possuem efeito antirradical livre, o que ativa o metabolismo energético tissular e melhora seu desempenho durante a hipóxia. Alguns trabalhos relatam melhora do desempenho mental com seu uso. Fazem parte deste grupo a pentoxifilina (reduz a viscosidade sanguínea e aumenta a deformidade eritrocitária, além de ter ação anti-inflamatória), a Gingko biloba (o extrato EGB 761 é o único com comprovável efeito) e o piracetan. Pacientes com problemas vasculares associados, especialmente idosos, são os mais beneficiados com sua utilização. Como efeitos colaterais, a pentoxifilina pode desencadear rubor facial e cefaleia.

Antidepressivos: indicados no tratamento da tontura crônica subjetiva, migrânea vestibular ou depressão associada ao quadro. São consideradas drogas facilitadoras da compensação central. Existem vários tipos que podem ser usados, e sua escolha é dada pelo perfil do paciente, associado aos possíveis efeitos colaterais, sendo os mais comuns o ganho de peso, cefaleia, ansiedade, redução da libido e outros, a depender da droga escolhida. Os antidepressivos mais frequentemente utilizados em Otoneurologia são a amitriptilina, a sertralina, a fluoxetina, a paroxetina, a venlafaxina e o escitalopram.

\section{Conclusões}

Podemos concluir, dessa forma, que a melhor droga a ser utilizada no tratamento da tontura de seu paciente é aquela que foi individualizada de acordo com o seu perfil e o momento da doença, sempre levando em consideração fatores como a patologia de base, idade, peso e comorbidades associadas. Não há fórmula-padrão para o tratamento da tontura. A medicação constitui, portanto, apenas uma das armas que temos para o tratamento, devendo outros fatores, como metabolismo, sono e cervical serem avaliados e tratados caso haja necessidade. 
Patrícia Mano • Abordagem e complicações medicamentosas na tontura

\section{Referências}

1. Leight J. Pharmacological and optical methods of treatment for vestibular disorder and nystagmus. In: Herdman SJ, editor. Vestibular Rehabilitation. Philadelphia: F. A. Davis Company; 2007. p. 178-187.

2. Curthoys IS, Halmagyi GM. Vestibular compensation: clinical changes in vestibular function with time after unilateral vestibular loss. In: Herdman SJ, editor. Vestibular Rehabilitation. F. A. Davis Company: Philadelphia; 2007. p.76-97.
3. Shepard NT. Medical therapy for the balance disorder patient. In: Shepard NT, Telian AS, editors. Pratical manangement of the balance disorder patient. Singular Publishing Group: California; 1996. p. 187-192.

4. Baloh RW. The peripheral vestibular system. In: Baloh RW, Kerber KA, Clinical neurophysiology of the vestibular system. 4th ed. Oxford University Press: New York; 2011. p.25-62.

5. Baloh RW. Symptomatic treatment of vertigo. In: Baloh RW, Kerber KA, Clinical neurophysiology of the vestibular system. 4th ed. Oxford University Press: New York; 2011. p.405-418. 